\title{
Early referral to palliative care in IPF - pitfalls and opportunities in clinical trials
}

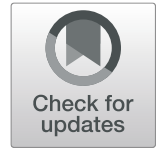

Meena Kalluri ${ }^{1^{*}}$ (D, Elisabeth Bendstrup ${ }^{2}$, Kathleen O. Lindell ${ }^{3}$ and Giovanni Ferrara ${ }^{4}$

Keywords: IPF, Palliative care, Early referral to palliative care

\section{Letter to the editor}

To the Editors,

BMC Respiratory Research

Dear Drs. Bals and Tantisira,

We read with great interest the study by Janssen et al. to assess feasibility of measuring the effect of a palliative care (PC) clinic referral on quality of life, anxiety and depression in Idiopathic Pulmonary Fibrosis (IPF) [1]. We would like to congratulate the authors for undertaking research in the much-needed area of IPF palliative care. The study was a pilot study and as such not powered to detect impact of intervention. The authors succeeded in demonstrating that this type of study is feasible but the intervention (referral to PC clinic) did not show any impact on health related quality of life (HrQoL) as measured by St. George respiratory Questionnaire (SGRQ) total score, and anxiety and depression (HADS) at 6 months. At 3 months follow up, SGRQ symptoms score and depression showed transient worsening in the intervention group.

In any clinical trial, the goals are to recruit the target population without significant bias, apply standardized interventions and use the best measurement tool to detect the effect of interventions. The authors highlight to several important poinst to consider in understanding the results. First, selection bias may have limited the enrolment into the study. Off 55 eligible patients, only 22 (40\%) consented to participate. This is in sharp contrast to recent trials in IPF $[2,3]$ where all eligible patients

\footnotetext{
* Correspondence: Kalluri@ualberta.ca

${ }^{1}$ Division of Pulmonary Medicine, Department of Medicine, University of Alberta, Alberta Health Services, Edmonton, Canada

Full list of author information is available at the end of the article
}

meeting inclusion criteria were easily enrolled. The authors correctly note that preconceived ideas of PC may have negatively influenced enrollment. On the other hand, two patients declined to be randomized and wanted to receive palliative care outright. This demonstrates the wide variability in understanding and acceptability of PC within this population. IPF patient and caregiver perceptions of $\mathrm{PC}$ are frequently negative at the outset $[4,5]$. We believe that this perception, in addition to the design of the study, may have influenced acceptance, adherence to and ultimately success of PC interventions.

In the AmbOx study, 33\% of interviewed patients who had negative perceptions of oxygen did not value this therapy and hence chose to discontinue oxygen despite perceived improvement of their symptoms [6]. We suspect this may also be true with PC. If recruited patients have a negative perception of the intervention, it is likely that they may not perceive value despite benefit, and will be less likely to accept and continue therapies, impairing the overall results of the study. While no qualitative analysis was performed in this study, the informal narrative comments do reflect this concern. Some patients found PC beneficial and others did not, these individuals did not value the consultation at all and could not recall the visit. This patient characteristic may have a profound impact on the results and its magnitude outside the intrinsic merits of the intervention itself. Increased understanding of the impact of patient perceptions on acceptance, adherence to interventions and self-reported research outcomes will lead to better study design. It may be important to stratify patients based on this characteristic, a perception and personality informed cohort enrichment [7]. 
Palliative care is a complex intervention with several different components; where the delivery of each component depends on individual patient's needs that vary across the disease trajectory [8]. Hence, PC requires individualization, unlike a "pill" and presents challenges to clinical trial design given the heterogeneity in the disease, patient needs and the clinical approach. IPF patients present several unique challenges: some patients and their families' desire practical advice on how to live well (better treatment of symptoms, improved self-management efficacy etc), others want practitioners to emphasize hope and not just provide information about disease and its management [9]. Some want open and honest discussions on end of life such as how death looks like, how to prepare for the future and more psychosocial support [9-11]. There are even differential information needs between patients and their families based on severity of disease and their own personalities [12, 13]. As patients and families adapt to living with the diagnosis they may move through various phases of shock, denial or acceptance and positive or maladaptive coping [14] depending on their personalities. Future trials will have to consider such adaptive responses that will evolve with disease and time, and design appropriately tailored PC interventions for such a broad range of needs.

How to identify and address these unique needs in clinical practice is the subject of ongoing research. There are several trials underway focused on the various PC components in IPF [15-17]. We hope that these results will highlight the complexity of PC interventions for IPF patients and inform intervention choice in future trials. Specialized research designs such as the sequential multiple assignment-randomized trial (SMART) maybe useful to study such complex PC interventions [18]. Until such time, it may be useful for PC clinical trials to document the actual needs as stated by patients and the individualized interventions applied. Janssen et al. indicate that pharmaceutical interventions were employed in only 2 patients and the majority presumably received education, information or support through the multidisciplinary palliative team. This suggests that most patients' needs were educational. They do not report if clear practical advice on other aspects of symptom management (e.g. oxygen titrations, pacing, activity modification etc) was delivered. Patients' needs or goals are not clearly documented at enrolment and therefore, it is uncertain if they were met. In such cases, it is possible that patients may not perceive any tangible benefits as interventions (discussions and actions) were not targeted to needs.

We agree with the authors that discussions regarding prognosis and Advanced Care Planning (ACP) can provoke anxiety if not balanced with practical advice perceived as beneficial and hopeful by the patient. Pooler et al. showed that in the long term, however, $\mathrm{ACP}$ and other discussions were perceived to be beneficial by bereaved IPF caregivers. Some subjects reported initial anxiety, but in retrospect, they agreed that it was beneficial to "know" and be prepared for the future (Pooler [19]). While confirmation in prospective studies is needed, it may be important for clinicians to know that short-term anxiety can occur but in the long term, such discussions may have tangible longer-term benefits such as decrease in ineffective and dangerous therapies, and reduced end of life hospitalizations [20]. This poses another problem when designing clinical trials for PC interventions, as the duration of the trial can have a great impact on the measured results and on their interpretation. We also agree that the delivery of such complex PC interventions may need longitudinal support both inside and outside clinics, and involve the care givers [8, 21-24]. A clinicbased intervention while useful may not be able to fully address all needs as disease progresses [9, 23].

Lastly, what is the right measurement tool for quality of life in IPF? HrQoL is a self-report of patient perception of their health status. It is captured by using tools called patient reported outcome measure (PROM). While there are several well validated and frequently used PROM in IPF [25] very few meet all the standards proposed by the Food and Drug Administration (FDA) [26]. The authors used SGRQ in this study; it was originally developed for COPD population, although validated later for IPF. There are items in the SGRQ symptom domain that are not relevant for this disease. Amongst all the available tools, only SGRQ- I and the Kings-brief interstitial lung disease (K-BILD) questionnaire are disease specific and concordant with FDA recommendations for PROM use in clinical trials [27-29]. Even so, K-BILD does not measure cough, a very important and burdensome symptom in this population [30]. This highlights the challenge of finding the right tool to measure HRQol in clinical trials. In the AmbOx study, K-BILD symptom score registered improvement in dyspnea that was not detected by the SGRQ symptom domain. This suggests that the use of disease specific PROM may yield more accurate results and with greater fidelity than a nonspecific PROM. Given the variability in needs based on disease stage, computerized adaptive designs for PROM may be useful rather than using paper based SGRQ that can take approximately $1 \mathrm{~h}$ to complete and is also burdensome by patient report in this study. While PROM are essential to measure impact on patients as perceived by them, other outcomes are also equally important when reviewing PC interventions. For example, the authors highlight that one patient was referred to hospice because of the PC referral: This is essential to improve end of life care and hence a vital and important outcome by itself [31]. 
Future work in this field can build further on Janssen et al's research. We look forward to a new era of clinical trials where patient centered interventions like palliative care will be assessed in appropriately designed trials, using disease specific PROMs to build the needed evidence base to demonstrate the intrinsic value of palliative medicine for ILD patients.

\section{Abbreviations}

IPF: Idiopathic Pulmonary Fibrosis; PC: Palliative care; HrQoL: Health related quality of life; SGRQ: St. George respiratory Questionnaire (SGRQ) total score; HADS: Hospital anxiety and depression questionnaire; AmbOx: Ambulatory oxygen; SMART: Sequential multiple assignment-randomized trial; ACP: Advanced Care Planning; PROM: Patient reported outcomes measure; FDA: Federal Drug Administration; COPD: Chronic obstructive pulmonary disease; SGRQ- I: St. George respiratory Questionnaire for IPF; K-BILD: Kingsbrief interstitial lung disease questionnaire; ILD: Interstitial lung disease

\section{Acknowledgements}

Not applicable.

\section{Authors' contributions}

MK: drafted the letter, edited and reviewed the final submission. EB: provided content input, critical feedback, edited and reviewed the final submission. KL: provided content input, critical feedback, edited and reviewed the final submission. GF: provided content input, critical feedback, edited and reviewed the final submission. The author(s) read and approved the final manuscript.

\section{Funding}

None.

Availability of data and materials

Not applicable.

\section{Ethics approval}

Not applicable.

\section{Consent for publication}

Not applicable.

\section{Competing interests}

MK: no funding to declare or any conflicts of interest related to this work. EB: no funding to declare or any conflicts of interest related to this topic. KL: reports National Institute of Nursing Research funding for palliative care clinical trial; COI: Genentech ILD Nursing Advisory Board.

GF: no funding to declare or any conflicts of interest related to this topic.

\section{Author details}

'Division of Pulmonary Medicine, Department of Medicine, University of Alberta, Alberta Health Services, Edmonton, Canada. ${ }^{2}$ Center for Rare Lung Diseases, Department of Respiratory Diseases and Allergy, Aarhus University Hospital, Aarhus, Denmark. ${ }^{3}$ University of Pittsburgh Dorothy P. \& Richard P. Simmons Center for Interstitial Lung Disease at UPMC, Pittsburgh, PA, Division of Pulmonary, Allergy, and Critical Care Medicine, Pittsburgh, PA, USA. ${ }^{4}$ Division of Pulmonary Medicine, Department of Medicine, University of Alberta, Alberta health Services, Edmonton, AB, Canada.

Received: 29 May 2020 Accepted: 8 June 2020

Published online: 08 July 2020

\section{References}

1. Janssen K, Rosielle D, Wang Q, Kim HJ. The impact of palliative care on quality of life, anxiety, and depression in idiopathic pulmonary fibrosis: a randomized controlled pilot study. Respir Res. 2020;21(1):2.

2. Richeldi $L$, et al. Efficacy and safety of nintedanib in idiopathic pulmonary fibrosis. N Engl J Med. 2014;370(22):2071-82.

3. King $T E$, et al. A phase 3 trial of Pirfenidone in patients with idiopathic pulmonary fibrosis. N Engl J Med. 2014;370(22):2083-92.
4. Lindell KO, Kavalieratos D, Gibson KF, Tycon L, Rosenzweig M. The palliative care needs of patients with idiopathic pulmonary fibrosis: a qualitative study of patients and family caregivers. Hear Lung J Acute Crit Care. 2017;46(1):24-9.

5. Kreuter $\mathrm{M}$, et al. Palliative care in interstitial lung disease: living well. Lancet Respir Med. 2017;5:968-80.

6. Visca D, et al. Effect of ambulatory oxygen on quality of life for patients with fibrotic lung disease (AmbOx): a prospective, open-label, mixed-method, crossover randomised controlled trial. Lancet Respir Med. Oct. 2018;6(10):759-70.

7. Oldham MA. Personality-informed care: speaking the language of personality. Psychosomatics. 2020;61:220-30.

8. Kalluri M, Luppi F, Ferrara G. What patients with idiopathic pulmonary fibrosis and caregivers want - filling the currents gaps with patient reported outcomes and experience measures. Am J Med. 2019;133(3):281-9.

9. Sampson C, Gill BH, Harrison NK, Nelson A, Byrne A. The care needs of patients with idiopathic pulmonary fibrosis and their carers (CaNoPy): results of a qualitative study. BMC Pulm Med. 2015;15(1):155.

10. Bajwah $\mathrm{S}$, et al. The palliative care needs for fibrotic interstitial lung disease: a qualitative study of patients, informal caregivers and health professionals. Palliat Med. 2013;27(9):869-76.

11. Holland $\mathrm{AE}$, et al. Be honest and help me prepare for the future: what people with interstitial lung disease want from education in pulmonary rehabilitation. Chron Respir Dis. 2015;12(2):93-101.

12. Ramadurai $\mathrm{D}$, et al. Understanding the informational needs of patients with IPF and their caregivers: 'You get diagnosed, and you ask this question right away, what does this mean? BMJ Open Qual. 2018;7(1):e000207.

13. Conoscenti CS, Rubin EM, Sapiro N. Patient journey with idiopathic pulmonary fibrosis (IPF): A breathtaking experience. Am J Respir Crit Care Med. 2013;187(4):2013.

14. Senanayake S, Harrison K, Lewis M, McNarry M, Hudson J. Patients' experiences of coping with idiopathic pulmonary fibrosis and their recommendations for its clinical management. PLoS One. 2018;13:e0197660.

15. Lindell $\mathrm{KO}$, et al. Randomised clinical trial of an early palliative care intervention (SUPPORT) for patients with idiopathic pulmonary fibrosis (IPF) and their caregivers: protocol and key design considerations. BMJ Open Respir Res. 2018;5:e000272.

16. Wright $\mathrm{C}$, et al. A feasibility, randomised controlled trial of a complex breathlessness intervention in idiopathic pulmonary fibrosis (BREEZE-IPF): study protocol. ERJ Open Res. 2019:5(4):00186-2019.

17. "Nebulized Fentanyl in Patients With Mild to Moderate Idiopathic Pulmonary Fibrosis and Chronic Dyspnea - Tabular View - ClinicalTrials.gov." [Online]. Available: https://clinicaltrials.gov/ct2/show/record/NCT03018756 ?term $=$ dyspnea\&cond=IPF\&draw $=2 \&$ rank $=3$. [Accessed: 18-Feb-2020].

18. Almirall D, Nahum-Shani I, Sherwood NE, Murphy SA. Introduction to SMART designs for the development of adaptive interventions: with application to weight loss research. Transl Behav Med. 2014;4(3):260-74.

19. Early integrated palliative appraoch for idiopathic pulmonary fibrosis: A narrative study of bereaved caregivers' experiences. Palliat Med 2018;32(9): 1455-1464.

20. Kalluri M, Lu-Song J, Younus S, Nabipoor M, Richman-Eisenstat J, Ohinmaa A, Bakal JA. Health Care Costs at the End of Life for Patients with Idiopathic Pulmonary Fibrosis. Evaluation of a pilot multidisciplinary collaborative interstitial lung disease clinic. Ann Am Thorac Soc. 2020;17(6):706-13.

21. Kalluri M, Richman-Eisenstat J. Early and integrated palliative care to achieve a home death in idiopathic pulmonary fibrosis. J Pain Symptom Manage. 2017;53(6):1111-5

22. Kalluri M, Richman-Eisenstat J. Breathing is not an option; dyspnea is. J Palliat Care. 2014;30(3):188-91.

23. Bajwah S, et al. Palliative care for patients with advanced fibrotic lung disease: a randomised controlled phase II and feasibility trial of a community case conference intervention. Thorax. Sep. 2015;70(9):830-9.

24. Kalluri M, Claveria F, Ainsley E, Haggag M, Armijo-Olivo S, Richman-Eisenstat J. Beyond idiopathic pulmonary fibrosis diagnosis: multidisciplinary care with an early integrated palliative approach is associated with a decrease in acute care utilization and hospital deaths. J Pain Symptom Manage. 2018; 55(2):420-6.

25. Wijsenbeek M, Van Manena M, Bonella F. New insights on patient-reported outcome measures in idiopathic pulmonary fibrosis: Only PROMises? Curr Opin Pulm Med. 2016;22:434-41.

26. FDA, "Guidance for Industry Patient-Reported Outcome Measures: Use in Medical Product Development to Support Labeling Claims," 2009. 
27. Belkin A, Albright K, Swigris JJ. A qualitative study of informal caregivers' perspectives on the effects of idiopathic pulmonary fibrosis. BMJ Open Respir Res. 2014;1:e000007.

28. Swigris JJ, et al. Patients' perceptions and patient-reported outcomes in progressive-fibrosing interstitial lung diseases. Eur Respir Rev. 2018;27(150):180075.

29. Moor CC, Heukels P, Kool M, Wijsenbeek MS. Integrating patient perspectives into personalized medicine in idiopathic pulmonary fibrosis. Front Med. 2017:4:226.

30. Patel AS, et al. The development and validation of the King's brief interstitial lung disease (K-BILD) health status questionnaire. Thorax. 2012;67(9):804-10.

31. Archibald N, Bakal JA, Richman-Eisenstat J, Kalluri M. Early Integrated

Palliative Care Bundle Impacts Location of Death in interstitial lung disease: a pilot retrospective study. Am J Hosp Palliat Care. 2020. https://doi.org/10. 1177/1049909120924995.

\section{Publisher's Note}

Springer Nature remains neutral with regard to jurisdictional claims in published maps and institutional affiliations.

Ready to submit your research? Choose BMC and benefit from:

- fast, convenient online submission

- thorough peer review by experienced researchers in your field

- rapid publication on acceptance

- support for research data, including large and complex data types

- gold Open Access which fosters wider collaboration and increased citations

- maximum visibility for your research: over $100 \mathrm{M}$ website views per year

At BMC, research is always in progress.

Learn more biomedcentral.com/submissions 